\title{
Modified model of porosity variation in seepage fluid-saturated porous media under elastic wave
}

\author{
Liming Zheng ${ }^{1} \cdot$ Chunsheng Pu ${ }^{1,2} \cdot$ Jiaxiang $\mathrm{Xu}^{1} \cdot$ Jing Liu $^{1} \cdot$ Xiaolin Zhao $^{3}$
}

Received: 8 August 2015/ Accepted: 25 November 2015/Published online: 17 December 2015

(C) The Author(s) 2015. This article is published with open access at Springerlink.com

\begin{abstract}
As an unconventional method, low-frequency vibration production technology is applied for oil recovery enhancement in amounts of oil fields. Petro-physic properties in seepage fluid-saturated porous media with are revealed to be influenced seriously by elastic wave in substantial researches. With an assumption of static fluidsaturated, porosity variation equation in Biot's model is not appropriate to interpret the percolating in low-frequency vibration production technology, there exists an obvious error from the actual experimental data. Based on the function mechanisms and factors controlling the porosity in seepage fluid-saturated porous media, a modified model of porosity variation under vibration is developed to improve the accuracy. Different coefficients for porosity increase are derived. The factors include the compaction of matrix and fluid, proportion of seepage interconnected pores, change of adhesive layer in capillary tube as well as transport of particle under vibration. Furthermore, a comparison with experimental result in artificial sandstone cores is carried out to verify the validity. An error of about $4-21 \%$ is reduced by the modified porosity variation compared with that in Biot's model. As a critical basis of wave equation, the modified porosity is helpful to illustrate the concrete change of percolation in low-frequency vibration production technology.
\end{abstract}

Liming Zheng

upczlm@sina.cn; zheng_liming_happy@126.com

1 College of Petroleum Engineering, China University of Petroleum (Eastern China), No. 66 Yangtze River West Road, Huang Dao District, Qingdao, China

2 State Key Laboratory of Heavy Oil, Qingdao, China

3 Logging Co. Ltd. of GWDC, Panjin, China
Keywords Modified model · Porosity variation - Seepage fluid - Elastic wave $\cdot$ Low-frequency vibration production technology

\section{Introduction}

The low-frequency vibration production technology, called also (artificial) seismic production technology, has been trialed in many oil fields. It is an alternative method to enhance oil recovery. Many researches are conducted on its principles, appliances and production improvement with vibration stimulation. A new research direction, as vibration-assisted chemical flooding technology, is also extended. However, the progress above still cannot meet the demand at the scene and the effects for production increase are far from satisfactory. The fundamental seepage theory for low-frequency vibration production technology is also still inadequate, which may be a possible explanation for certain failures unexpected in trial tests. It is highly necessary to develop a new seepage model and carry out studies on seepage change or light oil redistribution in fluid-saturated media under elastic wave.

Certainly, change in physical properties under elastic wave should be clarified firstly, as a preliminary basis of above researches. The physical properties include fluid viscosity, porosity, elastic moduli of solid and fluid phases, permeability, pore structure, propagation speed and attenuation of wave and so on. Much of them are already investigated through experiments and described with expressions. But some are still instinctive or unsuitable with the expressions defined, such as the viscosity and porosity, with less consideration on the impacting factors and function mechanisms in low-frequency vibration production technology. Therefore, it is necessary to improve 
the equation and verify the validity of a new model compared with the experimental data, which is the main work in this paper.

Generally, there are several kinds of equations connected with porosity variation under elastic wave. The first one is based on the Wyllie equation and used in interpretation of acoustic logging (Zhang et al. 2009).The second kind is the equations applied currently in petro-physics and seismic exploration (Shi et al. 2014; Mavko et al. 1998; Wang and Zhong 2012; Kuster and Toksoz 1974). Results about the elastic modulus are involved in the papers of Gassmann, Mavko, Biot, Dvorkin, Sun as well as the porosity model of Kuster-Toksöz, Xu-White, etc. The equations are derived from theoretical hypothesis or experimental measurements in unsaturated, partial-saturated or saturated porous media with or without inner seepage flow. Making using of the concrete stress-strain change in situ under elastic wave and the relations of physical parameters and strain, the third kind equation about porosity variation can be derived. Assuming the solid unit displacement is $u=A_{0} \sin (\omega t-l r)$ and the additional pressure posed on porous media by the elastic wave is $P_{1}=P_{p}+\rho C A_{0} \omega \cos (\omega t-l r)$ (Luo et al. 1996; Liu et al. 2011). Then the additional stress caused by vibration can be calculated with the stress model of Biot theory (Ge et al. 1994). With the constitutive equation $\sigma_{i j}=C_{i j k l} e_{k l}$, a new value of strain can be obtained. Ignoring the volume change of solid grain and cement and combining the porosity-strain relations $\phi=1-\frac{1-\phi_{0}}{1+\varepsilon_{v}}\left(1+\frac{\Delta V_{s}}{V_{s 0}}\right)$, the bulk strain variation, its maximum value, and derivative over time under vibration or not could be derived $(\mathrm{Li}$ et al. 2003). However, with the assumption of given stress change and static fluid saturation, there exists a margin of error between the third method and the actual porosity variation in seepage media.

However, the micro-active mechanisms causing the porosity change in rocks saturated with percolating fluid under vibration is not considered in above models. The actual effective porosity in seepage media under vibration is different from that with initial static fluid saturated under vibration, or that with seepage fluid saturated with no wave impacting. It is necessary to consider the factors impacting the porosity in low-frequency vibration production technology, which is proven by experiments.

Low-frequency vibration production technology could improve the oil recovery through the follow mechanisms (Cidoncha 2007; Ariadji 2005; Kurawle et al. 2009): (1) reducing the capillary forces and fine particles blocking rate inner pore throats could increase the absolute permeability of rocks; (2) crude oil viscosity decrease, hydrophilicity increase on rock surface, and an additional drive force added by vibration could improve the fluid seepage rate; (3) velocity difference between solid and fluid might increase crude oil detachment or extraction in bypassed throats, so as to reduce residual oil saturation and improve oil recovery. Thereby, the real pore volume under wave is only part of the whole pores. On account of the mechanisms above, many factors like the high interface tension in micro- or ultra-micro-fine pores and throats, adhesive layers along fluid pass away, pore volume variation by the compressible rock and fluid, particle migration, and crack extending, might cause a shift in the value of effective porosity under elastic wave. Based on the factors above, the modified porosity variation equation is established.

\section{Modified effective porosity variation in seepage media}

Based on finite deformation of elastic solids and the Biot poroelastic theory, it is assumed that: (a) fluid-solid coupling is neglected when without vibration; (b) elasticplastic property of rock is not affected by low-frequency vibration production technology, with the porous media keeping isotropic and elastic; (c) the Poiseuille flow of the compressible fluid is kept under certain frequency, which is less than the characteristic frequency $\omega_{c}$; (d) the pore size is much smaller than the wave length and the characteristic length of the reservoir.

Under elastic wave, the effective porosity variation in seepage media can be expressed as a multiple of that in static fluid-saturated media as Eq. 1. The coefficient $\xi$ is an increment (Eq. 2) which integrates the amount of mobile pores, actual percolating space per pore/throats, as well as the additional change in pore volume by different packing mode and fracture distribution. Certainly, the porosity variation by the packing mode in rocks cemented closely, and that by the fracture redistribution in single-porosity media or in dual-porosity media with high crack threshold pressure, can be ignored.

$d \phi=d \phi_{v} \cdot \xi$

$\xi=\xi_{a} \cdot \xi_{c} \cdot \xi_{p}$

where $d \phi$ is the effective porosity variation in seepage media under wave; $d \phi_{v}$ is the porosity variation due to the change of rock and fluid compressibility; $\xi_{a}, \xi_{c}$ and $\xi_{p}$ are the coefficients of a value larger than 1 that demonstrates the influence of elastic wave on, respectively, the adhesive 
layer, capillary force and particle transport in seepage media.

The porosity variation due to compaction of the matrix and fluid can be described by the relationship of deformation, porosity, and porous pressure in Biot poroelastic theory, in which the wave propagates in initial static fluidsaturated media (Dvorkin and Nur 1993; Biot 1956).

$d \phi_{v}=\alpha d e+\frac{d P_{f}}{Q}$,

where $\alpha$ is the Biot coefficient; $e$ is the strain of matrix; $1 / Q$ is the attenuation factor; $d P_{f}$ is the porous pressure changes, derived by the calculated porous pressure at $t+1$ under vibration minus that at a previous time $t$.

The porous pressure under vibration is a major dependent variable in terms of solid-fluid displacements in general Biot's consolidation problem under initial petrophysical properties. It is used to obtain the new values of petro-physical properties, including the actual porosity related with this paper, as well as the permeability and elasticity modulus of matrix which are functions of the porosity. The general iterative sequence makes sense of the porous pressures under different condition in following sections. And it enables the invocation of the porous pressures under different times promptly.

\section{Variation in the proportion of seepage interconnected pores}

Under elastic wave stimulation, the proportion of seepage interconnected pores is changed, which is reflected by the shape of capillary curve and relative permeability curve. The average throat radius is generally increased and of a higher-concentrated distribution. To describe the pore connectivity more directly, the coefficient is expressed as a function of porous pressure before or after vibration which is related to the throat radius (Tiab and Donaldson 2003; Hamida and Babadagli 2006; Namba and Hiraoka 1995), $\xi_{c}=\xi_{c}\left(P_{f}, P_{f f}\right)$.

The distribution curve of pore and throat under different capillary force is

$\int_{r_{\max }}^{r_{\min }} \Delta S_{\mathrm{Hg}} d(\log r)=1-S_{c}$

where $S_{\mathrm{Hg}}$ is the mercury saturation in pressured-mercury testing; $r_{\min }$ and $r_{\max }$ are the upper and lower limit of porethroat radius, respectively; $S_{c}$ is the minimum saturation of wetting phase (corresponding to the irreducible water saturation of rock).

Assuming a lognormal distribution for the distribution of pore-throat size, with the average value of $\log r_{d 50}$ (the large distribution domain of median pore-throat radius corresponding to the median saturation pressure) and scale parameter of $\sigma, \Delta S_{\mathrm{Hg}}$ is expressed as below

$$
\Delta S_{\mathrm{Hg}}=\frac{1}{\sqrt{2 \pi} \sigma\left(1-S_{c}\right)} \exp \left[-\frac{\left(\log r-\log r_{d 50}\right)^{2}}{2 \sigma^{2}}\right] \text {. }
$$

When $\sigma=1, \Delta S_{\mathrm{Hg}}=\frac{1}{\sqrt{2 \pi}\left(1-S_{c}\right)} \exp \left\{-\frac{\left[\log \left(r / r_{d 50}\right)\right]^{2}}{2}\right\}$.

With $r=\frac{2 \sigma_{c} \cos \theta}{P_{c}}$, Eq. 4 can be expressed as $\int_{0}^{P_{f} \lim } \Delta S_{\mathrm{Hg}} d\left(\log \frac{2 \sigma_{c} \cos \theta}{P_{f}}\right)=1-S_{c}$, when the capillary force $P_{c}$ equals to the given porous pressure $P_{f}$ in single fluid-saturated media. The minimum throat size $r_{\min }$ is corresponding to the fluid pressure $P_{f \lim }$, whereas the maximum throat size $r_{\max }$ is corresponding to the threshold pressure $P_{f \max } \rightarrow 0$. Then, the porosity $\phi_{2}$ occupied by the fluid with a displacement pressure $P_{f}$ is as below, taking the location parameter of $\log r_{d 50}$ and scale parameter of 1 into account (Fig. 1).

$$
\begin{aligned}
\phi_{2}= & \int_{r_{\max }}^{r} \Delta S_{\mathrm{Hg}} d(\log r)=1-S_{c}-\int_{r}^{r_{\min }} \Delta S_{\mathrm{Hg}} d(\log r) \\
= & \int_{0}^{P_{f}} \frac{1}{\sqrt{2 \pi}\left(1-S_{c}\right)} \exp \left\{-\frac{1}{2}\left[\log \left(\frac{2 \sigma_{c} \cos \theta}{P_{f} r_{d 50}}\right)\right]^{2}\right\} \\
& \times d\left(\log \frac{2 \sigma_{c} \cos \theta}{P_{f}}\right) \\
= & \frac{1}{\sqrt{2 \pi}\left(1-S_{c}\right)} \int_{2 \sigma_{c} \cos \theta / r_{d 50} \cdot \exp (-y)}^{+\infty} \exp \left(-\frac{1}{2} y^{2}\right) \mathrm{d} y \\
= & \frac{1}{\sqrt{2 \pi}\left(1-S_{c}\right)}\left[\frac{\sqrt{\pi}}{2}-\Phi\left(P_{f} ; \log r_{d 50}, 1\right)\right],
\end{aligned}
$$

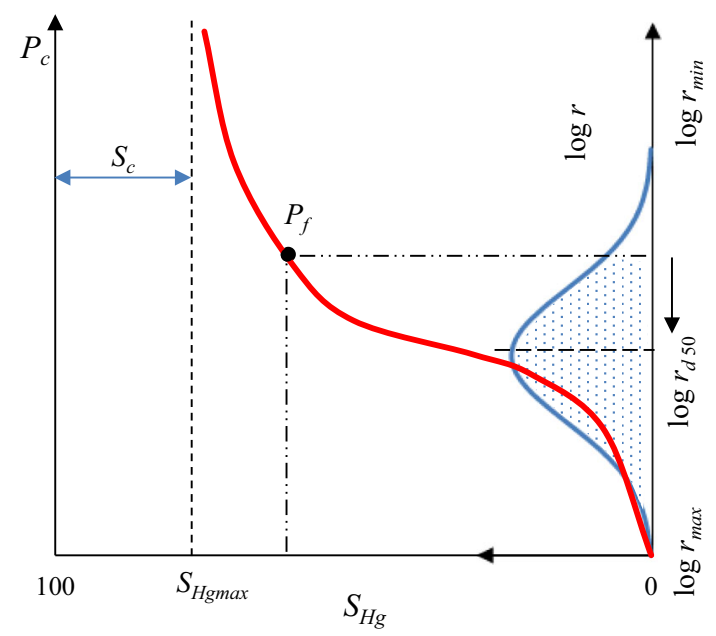

Fig. 1 Typical capillary curve and lognormal distribution of porethroat size 
where $\Phi\left(P_{f} ; \log r_{d 50}, 1\right)$ is the cumulative normal distribution function; $\sigma_{c}$ is the interfacial tension between the wetting phase and the non-wetting phase; $\theta$ is the contact angle.

Thereby, the coefficient for porosity variation under elastic wave due to the change of capillary curve is as Eq. 7.

$\xi_{c}=\frac{\int_{0}^{P_{f}} \Delta S_{\mathrm{Hg}} \mathrm{d} r}{\int_{0}^{P_{f f}} \Delta S_{\mathrm{Hg}} \mathrm{d} r}=\frac{\frac{\sqrt{\pi}}{2}-\Phi\left(P_{f} ; \log r_{d 50}, 1\right)}{\frac{\sqrt{\pi}}{2}-\Phi\left(P_{f f} ; \log r_{d 50}, 1\right)}$,

where the subscripts $f$ and $f f$ represent the physical property in seepage media with or without vibration stimulation, respectively; $P_{f}$ is the porous pressure under vibration; $P_{f f}$ is the original porous pressure with no vibration impacting.

Certainly, based on the corresponding relation on $P_{c} \sim S_{\mathrm{Hg}}, \xi_{c}=\frac{S_{\mathrm{Hg}}\left(P_{f}\right)}{S_{\mathrm{Hg}}\left(P_{f f}\right)}$ when $S_{\mathrm{Hg}}<S_{\mathrm{Hgmax}}$, and $\xi_{c}$ is close to 1 when $S_{\mathrm{Hg}} \geq S_{\text {Hgmax }}$.

\section{Change of adhesive layer in capillary model}

The increased volume of seepage interconnected pores in mercury-displaced rock can be seen as the increased tortuosity in a single capillary model. Whereas, the flowing radius and thickness of adhesive layer determine the porosity variation coefficient $\xi_{a}$ in a constant-radius percolating tube. And the flowing radius is seriously influenced by the fluid viscosity and relative motion of solidfluid phase (Wei 2010; Antara and Demiray 2000). Thereby, $\xi_{a}$ can be expressed as a function of $\bar{U}-u$ and $\eta$. According to the Prandtl's adhesion layer theory, it is assumed that the flow outside the narrow region adjacent to the fixed area boundary is reckoned as an ideal fluid flow, whereas the viscous force dominates the flow inside the

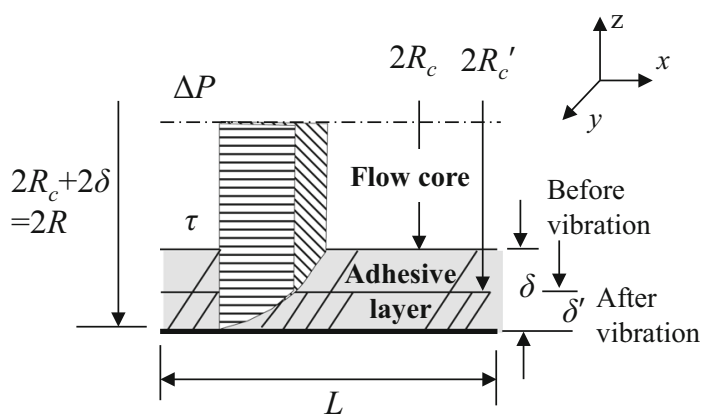

Fig. 2 Diagram of adhesive layer in half section of pipe flow narrow area adjacent to the fixed area boundary. Towards the fluid of high viscosity, the impact of slip effect between solid-fluid under vibration on porosity variation cannot be neglected, with the decrease of fluid shear force and adhesive layer thickness.

In the capillary flow model of length $L$ and diameter of $R$, it is assumed that the adhesive layer in Fig. 2 influenced by viscous resistance is close to immobile and with a thickness of $\delta$ under certain drive pressure of $\Delta P$. The radius for actual pipe flow in the center is $R^{\prime}=R-\delta$. In the area packed with horizontal line and diagonal, the interface shear force acting on the fluid decrease gradually. When the force is small enough, the outermost fluid in the flow core begin to move. If a vibration is imposed on the model, the shear force on inner boundary of adhesion layer varies, and the immobile flow is induced, represented as the bevel edge of the area packed with horizontal line. In this case, the radius of the pipe flow and thickness of the adhesive layer are affected.

When with non-influence of other external forces, the solid-liquid relative velocity in one-dimensional capillary flow under vibration is $U(x, r)=\frac{\partial(\bar{U}-u)}{\partial t}$. Then the differential equation for adhesive layer under vibration is as Eq. 8, with incompressible non-Newtonian fluid flow in the one-dimensional pipe. When $n=1$, Eq. 8 is simplified into the situation with Newtonian flow.

$$
\begin{array}{r}
-\frac{1}{\rho} \frac{\partial P_{f}}{\partial x}+\frac{\eta^{1 / n}}{\rho}\left(\frac{\partial^{2} U}{\partial r^{2}}+\frac{1}{r} \frac{\partial U}{\partial r}\right)^{1 / n}= \\
U_{x} \frac{\partial U_{x}}{\partial x}+U_{r} \frac{\partial U_{r}}{\partial r} \\
\frac{\partial U_{x}}{\partial x}+\frac{\partial U_{r}}{\partial r}=0
\end{array},
$$

where $U$ is the relative flow rate; $\bar{U}$ is the fluid displacement; $u$ is the solid displacement; $\rho$ is the fluid density; $r$ is the radius; $x$ is the axial distance along the tube; $n$ is the characteristic index of non-Newtonian fluid, with a pseudo-plastic style when $n>1$ or belonging to dilatant fluid when $n<1$.

Certainly, the parameters above are based on $\bar{U}=U_{f f}$, $u=0$ and $P_{f}=P_{f f}$ when there is no vibration impacting. According to previous research, the thickness of the adhesive layer can be expressed as a function of Reynolds number. For a non-Newtonian fluid, the thickness is also related to the characteristic index.

$\frac{\delta}{L}=A\left(\frac{1}{R e}\right)^{\frac{n}{1+n}}=A\left(\frac{K}{U^{2-n}(2 R)^{n} \rho}\right)^{\frac{n}{1+n}}$, 
where $A$ is a coefficient of different values for various types of fluids and flow patterns; Re is the Reynolds number, $R e=\frac{U^{2-n}(2 R)^{n} \rho}{K}$, and for a Newtonian fluid $n=1, K=\eta ; K$ is the consistency index.

Thereby, the coefficient $\xi_{a}$ for porosity variation under elastic wave due to the change of adhesive layer is as Eq. 10.

$$
\xi_{a}=\frac{(R-\delta)_{f}^{2}}{(R-\delta)_{f f}^{2}} \approx \frac{1-2 \delta_{f} / R}{1-2 \delta_{f f} / R}=\frac{1-\frac{2 A L}{R}(2 R)^{-\frac{n^{2}}{1+n}}\left(\frac{U_{f}^{2-n} \rho_{f}}{K_{f}}\right)^{\frac{-n}{1+n}}}{1-\frac{2 A L}{R}(2 R)^{-\frac{n^{2}}{1+n}}\left(\frac{U_{f f}^{2-n} \rho_{f f}}{K_{f f}}\right)^{\frac{-n}{1+n}}}
$$

Let $\frac{2 A L}{R}(2 R)^{-\frac{n^{2}}{1+n}}$ in a certain media be written as $\beta$, then the coefficient $\xi_{a}$ can be expressed as below, influenced by the flow rate, fluid density and the consistency index with different exponent

$$
\xi_{a} \approx \frac{1-\beta\left(U_{f}^{2-n} \rho_{f} / K_{f}\right)^{\frac{-n}{1+n}}}{1-\beta\left(U_{f f}^{2-n} \rho_{f f} / K_{f f}\right)^{\frac{-n}{1+n}}}
$$

\section{Transport of particles under the influence of elastic wave}

Under elastic wave stimulation, the particles in the pore and on the surface of matrix might be stripped down. A dynamic process of detachment, migration, deposition and new dispersion will be caused to a certain extent (Abbasi et al. 2011; Chun et al. 2011). When the particle, which is stripped down, flows out of a unit of porous media and comes into redistribution still inside the rock, the transport process of particle can be simplified. Consider only the proportion of pore throat for particulate clogging after particle migration, and neglect the redistribution of pore size and change in structure of pore throat after particle detachment and sedimentation. Then, the change rate of porosity can be indicated by the flow capacity $f$ of particles in throat. The flow capacity refers the ratio of channels allowable for particles migration to the total number of channels, based on the assumption of a stable lognormal distribution for the distribution of pore-throat size, $f=1-E_{r f} \int_{a_{p}}^{b_{p}} \frac{1}{\sqrt{2 \pi} S_{d} r \cdot \exp \left(\ln \sqrt{\gamma / d_{t}}-0.5 S_{d}\right)} \mathrm{d} r$

$\xi_{p}=\frac{f_{f}}{f_{f f}}$.

When the whole process of migration, deposition, and redistribution is considered after the particles flowing out of the matrix unit, porosity change due to particle transport

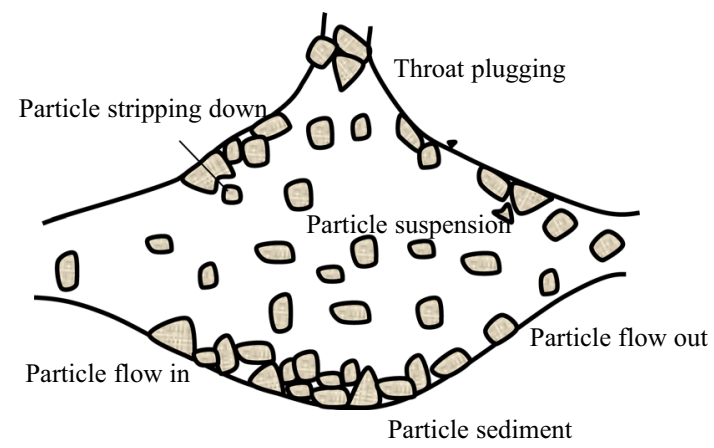

Fig. 3 Different particulate transport status in pore throat under vibration

under vibration is a sum of different transport rates (Bedrikovetsky et al. 2011; Rahman et al. 1994).

$\xi_{p}=1+\frac{\delta \phi}{\phi}=1+g_{b}-g_{d}-g_{p}$,

where $E_{r f}$ is the error function; $g_{b}$ is the rate for particle detachment from porous surfaces; $g_{d}$ is the net rate for particle sedimentation on porous surface; $g_{p}$ is the rate for throat plugging due to particle retention; $S_{d}$ is the geometry variance in pore distribution and with a value of 2 generally; $d_{t}$ is the equivalent throat width; $\gamma$ is the dimensionless pore-throat radius; $a_{p}$ is the minimum pore sizes; $b_{p}$ is the average particle diameter (Fig. 3).

\section{Equation for modified effective porosity variation}

Based on the analysis of above factors, the porosity variation in porous media saturated with seepage fluid can be obtained under the action of elastic waves

$$
\begin{aligned}
d \phi= & \left(\alpha d e+\frac{d P_{f}}{Q}\right) \cdot \frac{1-\beta\left(U_{f}^{2-n} \rho_{f} / K_{f}\right)^{\frac{-n}{1+n}}}{1-\beta\left(U_{f f}^{2-n} \rho_{f f} / K_{f f}\right)^{\frac{-n}{1+n}}} \\
& \cdot \frac{\frac{\sqrt{\pi}}{2}-\Phi\left(P_{f} ; \log r_{d 50}, 1\right)}{\frac{\sqrt{\pi}}{2}-\Phi\left(P_{f f} ; \log r_{d 50}, 1\right)} \cdot \frac{f_{f}}{f_{f f}} .
\end{aligned}
$$

To obtain the modified porosity variation, which is complex and of many unknown parameters, the calculation can be simplified as far as possible. Firstly, the fluid flow rate $U_{f}$, porous pressure $P_{f}$, and porosity variation $d \phi_{v}$ due to compressibility of matrix and fluid at time $t$ is obtained. Then, substitute $d \phi_{v}$ into the typical wave equation and get new values of rock physical property $P_{f}^{\prime}, U_{f}^{\prime}, \rho_{f}^{\prime}$ and $\eta_{f}^{\prime}$. Third, substitute the rock physical properties into the modified porosity model, and replace $d \phi_{v}$ with the new value of porosity variation $d \phi$. Finally, combined with $d \phi$ or $\xi$, establish the motion equation and continuity equation 
at the next moment in terms of the new porosity, $\phi_{t+1}=$ $\phi_{t}+d \phi_{t}$ or $\phi_{t+1}=\phi_{t}+\xi \cdot e_{t} \cdot \frac{1-\phi_{t}}{1+e_{t}}$. The vibration-induced physical property $P_{f}$ and $U_{f}$ at time $t+1$ can be solved subsequently. By repeating the process above, comparison of the physical properties under vibration or not is liable to illustrate the change in seepage characteristics and active mechanisms in the low-frequency vibration production technology.

\section{Comparison with the experimental data}

In comparison to the modified porosity variation, the experimental data of Ariadji (2005) are cited. The influence on crack and sand production by vibration in the closely cemented artificial cores is ignored. Combining the physical property change in T. Ariadji and the general elastic modulus of limestone or sandstone under elastic wave (Agersborg et al. 2008; Reine et al. 2012; Baechle et al. 2005), the ranges of different basic parameter in Eq. 14 are determined. The coefficients for matrix deformation and pressure change, $1 / Q$ and $\alpha$, are in the range of $0.0005-0.04$ and $0.90-0.95$, respectively. The results from the modified model, formula considering only the compressibility, and experiments are presented in Fig. 4.

By contrast, the increase rate of porosity from the Biot's model, reflecting compressibility of the matrix and fluid (mark as 'only consider compressibility'), is observed less than the experiment result. While the modified porosity variation is much close to the later, indicating that it is necessary to carry out the analysis. Define the error as the relative porosity increase rate of the calculated model from the experimental data before and after vibration, using one minus the ratio of different porosity increase rate. By comparison of three results, and subtracting the error of Biot's model from that of the modified model, it is found

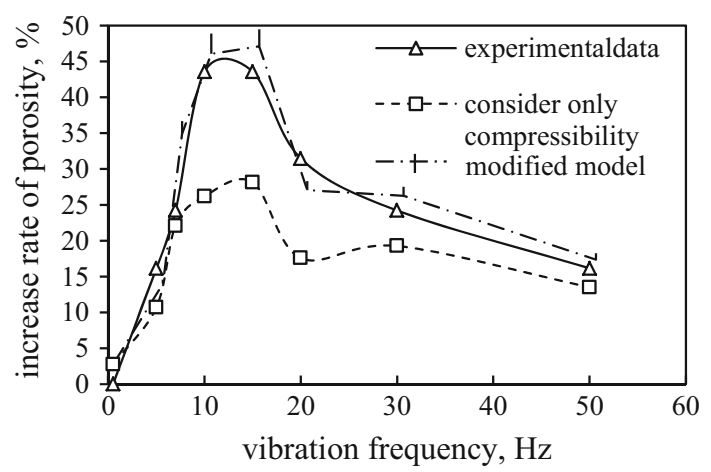

Fig. 4 Comparison of porosity variation in artificial core under different frequencies that the error is reduced for about 4-21\% generally. There is the most obvious amendment effect at the frequency $10-20 \mathrm{~Hz}$. When the frequency is minor $(5 \mathrm{~Hz})$, the experimental porosity changes slightly, closing to 0 , because of the measurement errors probably, while the porosity variation from the Biot's model and modified model is larger at $5 \mathrm{~Hz}$. Due to a large amount of action principles and parameters involved, the calculation in modified model is complex, with fluctuations existing in the data. Hence, the upper and lower limits of porosity variation are presented in the figure by a segment. At the place where the increase rate of porosity is larger, the fluctuation of numerical range becomes bigger. Certainly, there is still some error between the experiment data and the calculation result from the modified porosity model. Further modification for the model is needed, especially on the integration and simplification of the affecting factors, in order to facilitate the calculation. On one hand, the heterogeneity of interconnected pores in natural rock is stronger than that of the artificial cores which are cemented with sands of unified mesh size. There exist more fluctuations in the physical properties of natural rock under elastic wave. On the other hand, the elastic wave plays both promoting and inhibiting roles on the staggered movement of solid and seepage fluid in the porous media. When the inhibiting effect is stronger, the parameters of reservoir properties may be negative to the initial seepage. As a result, recognition and measurement of reservoir parameters under the action of elastic waves is more complicated and volatile.

\section{Conclusions}

A modified model of porosity variation under vibration is developed in this paper, based on the function mechanisms in low-frequency vibration production technology and factors impacting the porosity variation in seepage media. The factors include the amount of mobile pores, actual percolating space per pore/throats, as well as the additional change in pore volume by packing mode and fracture distribution. In contrast to the experimental data in artificial sandstone cores, the modified model of porosity variation reduces the error from that between the experiment data and the formula of Biot's model, which considers only the compaction of matrix and fluid. It is useful to illustrate the concrete change in the seepage under vibration, as a foundation of wave propagation in seepage media. Certainly, there is a necessity to further improve the model because of the error, which is mainly caused by the serious heterogeneity in natural rock and the double-sided roles, promoting and inhibiting, on the staggered solid-fluid motion. 
Acknowledgments Thanks to the National Natural Science Foundation of China (No. 51274229 and No. 2011ZX05009-004) for supporting our research on LF vibration oil extraction technology in low permeability reservoirs.

Open Access This article is distributed under the terms of the Creative Commons Attribution 4.0 International License (http:// creativecommons.org/licenses/by/4.0/), which permits unrestricted use, distribution, and reproduction in any medium, provided you give appropriate credit to the original author(s) and the source, provide a link to the Creative Commons license, and indicate if changes were made.

\section{References}

Abbasi S, Shahrabadi A, Golghanddashti H (2011) Experimental investigation of clay mineral' effects on the permeability reduction in water injection process in the oil fields [C]. SPE 144248

Agersborg R, Johansen TA, Jakobsen M et al (2008) Effects of fluids and dual-pore systems on pressure-dependent velocities and attenuations in carbonates [J]. Geophysics 73(5):35-47

Antara N, Demiray H (2000) The boundary layer approximation and nonlinear waves in elastic tubes $[\mathrm{J}]$. Int $\mathrm{J}$ Eng Sci 38(13):1441-1457

Ariadji T (2005) Effect of vibration on rock and fluid properties: on seeking the vibroseismic technology mechanisms [C]. SPE93112

Baechle GT, Weger RJ, Eberli GP et al (2005) Changes of shear moduli in carbonate rocks: implications for Gassmann applicability [J]. Lead Edge 24(5):507-510

Bedrikovetsky P, Siqueira FD, Furtado CA et al (2011) Modified particle detachment model for colloidal transport in porous media [J]. Transp Porous Media 86:353-383

Biot MA (1956) Theory of propagation of elastic waves in a fluidsaturated porous solid, II: low-frequency range [J]. J Acoust Soc Am 28(2): 168-178

Chun CS, Rao P, Xu HX et al (2011) Kinetic mechanism of inorganic scale plugging removal at near wellbore zone by high power ultrasonic technology [J]. J Chongqing Univ 34(4):47-52

Cidoncha JG (2007) Application of acoustic waves for reservoir stimulation [C]. SPE108643

Dvorkin J, Nur A (1993) Dynamic poroelasticity: a unified model with the squirt and the Biot mechanisms [J]. Geophysics 58(4):524-533
Ge HK, Huang RZ, Zhuang JJ et al (1994) Experimental study on dynamic and static elastic parameters of water-saturated sands under triaxial stresses [J]. J Univ Pet 3:41-47

Hamida T, Babadagli T (2006) Investigations on capillary and viscous displacement under ultrasonic waves $[\mathrm{J}]$. J Can Pet Technol 45(2): 16-19

Kurawle I, Kaul M, Mahalle N et al (2009) Seismic EOR-the optimization of aging waterflood reservoirs [C]. SPE123304

Kuster GT, Toksoz MN (1974) Velocity and attenuation of seismic waves in two phase media, Part I: theoretical formation [J]. Geophysics 39(5):587-606

Li W (2010) Wave surface of boundary layer [J]. Exp Thermal Fluid Sci 34(7):838-844

Li PC, Kong XY, Lu DT (2003) Mathematical modeling of flow in saturated porous media on account of fluid-structure coupling effect [J]. J Hydrodyn 18(4):419-426

Liu J, Pu CS, Liu T et al (2011) Study on the seepage law of formation fluid under the effect of pulse wave [J]. J Xi' an Shiyou Univ (Natural Science Edition) 26(4):46-49

Luo YT, Davidson B, Dusseault M (1996) Measurements in ultra-low permeability media with time-varying properties [C]. EUROCK1996-157

Mavko G, Mukerji T, Dvorkin J (1998) The rock physics handbook: tools for seismic analysis in porous media [M]. Cambridge University Press, New York

Namba T, Hiraoka T (1995) Capillary force barriers in a carbonate reservoir under waterflooding [C]. SPE-29773-MS

Rahman SS, Arshad A, Chen H (1994) Prediction of critical condition for fines migration in petroleum reservoirs [J]. SPE 28760

Reine C, Clark R, van der Baan M (2012) Robust prestack Q-determination using surface seismic data: part 1-method and synthetic examples [J]. Geophysics 77(1):45-56

Shi YM, Zhang Y, Yao FC et al (2014) Methodology of seismic imaging for hydrocarbon reservoirs based on acoustic full waveform inversion [J]. Chin J Geophys 2:607-617

Tiab D, Donaldson EC (2003) Petrophysics, Second Edition: Theory and Practice of Measuring Reservoir Rock and Fluid Transport Properties [M]. Houston, Tex: Gulf Pub. Print, 2003, pp 313-359

Wang P, Zhong GF (2012) Applications of rock physics models to the deep-sea sediment drift at ODP site 1144, northern South China Sea [J]. Adv Earth Sci 27(3):359-366

Zhang CG, Jiang WZ, Pan HP (2009) Principle and application of acoustic logging [M]. Petroleum Industry Press, Beijing 